\title{
Spectral Content Characterization for Efficient Image Detection Algorithm Design
}

\author{
Kyoung-Su Park, ${ }^{1}$ Sangjin Hong, ${ }^{1}$ Peom Park, ${ }^{2,3}$ and We-Duke Cho ${ }^{4}$ \\ ${ }^{1}$ Mobile Systems Design Laboratory, Department of Electrical and Computer Engineering, Stony Brook University - SUNY, \\ Stony Brook, NY 11794-2350, USA \\ ${ }^{2}$ Department of Industrial and Information Systems Engineering, Ajou University, Suwon-Si 442-749, South Korea \\ ${ }^{3}$ Humintec Co. Ltd., Suwon-Si 443-749, South Korea \\ ${ }^{4}$ Department of Electronics Engineering, College of Information Technology, Ajou University, Suwon-Si 442-749, South Korea
}

Received 8 August 2006; Revised 25 January 2007; Accepted 30 January 2007

Recommended by C.-C. Jay Kuo

\begin{abstract}
This paper presents spectral characterization for efficient image detection using hyperspectral processing techniques. We investigate the relationship between the number of used bands and the performance of the detection process in order to find the optimal number of band reductions. The band reduction significantly reduces computation and implementation complexity of the algorithms. Specifically, we define and characterize the contribution coefficient for each band. Based on the coefficients, we heuristically select the required minimum bands for the detection process. We have shown that the small number of bands is efficient for effective detection. The proposed algorithm is suitable for low-complexity and real-time applications.
\end{abstract}

Copyright (C) 2007 Kyoung-Su Park et al. This is an open access article distributed under the Creative Commons Attribution License, which permits unrestricted use, distribution, and reproduction in any medium, provided the original work is properly cited.

\section{INTRODUCTION}

The hyperspectral imaging systems have found various civilian and military applications. The high efficiency and flexibility of hyperspectral sensors provide a powerful measurement technology currently being demonstrated with modern airborne and spaceborne hyperspectral systems. The hyperspectral sensor typically gets one hundred to several hundreds of bands for exact spectral classification. The property of the hyperspectral sensor is similar to that of the sensor used in advanced digital cameras. The hyperspectral sensor is capable of covering infrared and/or ultraviolet radiation as well as visible light using the enormous number of bands; a typical digital camera sensor covers only visible light using three bands which are called RGB. The hyperspectral processing technology is gradually incorporated into modern civil and military remote sensing systems along with other sensors such as imaging radar and laser systems [1].

Hyperspectral processing requires an extremely large amount of input data for the spectral classification. Moreover, the computational requirement for processing input is significant. There are many approaches for analyzing hyperspectral data. Hardware clusters may be a feasible solution because they are used to achieve high performance, high availability, or horizontal scaling. Cluster technology can also be used for highly scalable storage or data management. These computing resources could be utilized to efficiently process the remotely sensed data before transmission to the ground [2]. Digital signal processors are also suitable for hyperspectral computations because it can be optimized for performing multiply-and-accumulate operations. It is usually implemented in digital signal processor (DSP) clusters for parallel processing $[1,2]$. Even though these processing systems have been applied for hyperspectral processing, high-speed image processing and efficient communication within processors are still hot issues. In addition, new processing algorithms and the highly effective memory management are essential for the new hyperspectral sensor which contains higher resolution and much more bands. For a realtime processing hyperspectral system, these are some of the key issues [3].

The objective of this paper is to characterize key parameters used in hyperspectral processing in order to minimize computational requirements, which are essential for high-speed real-time processing. Even though hyperspectral processing is often used in classification problems, we are 


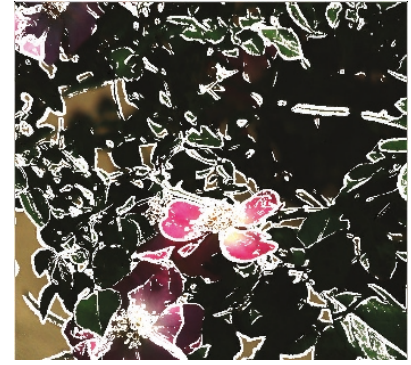

(a) Conventional

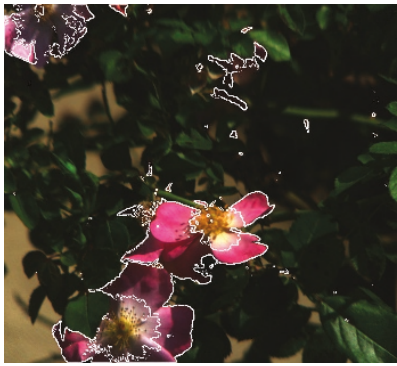

(b) Hyperspectral
Figure 1: Comparison of detected images based on conventional approach and hyperspectral approach.

focusing on target detection problems used in surveillance applications [4].

The rest of this paper is organized as follows. Section 2 describes the background of hyperspectral signal processing. The image data structures as well as processing data flow are described. We also characterize various key parameters involved in the detection process. Section 3 discusses detection characteristics as a function of the bands and libraries. In Section 4, we present a heuristic band selection strategy. The algorithm design and the evaluation are discussed in Section 5, and finally Section 6 concludes the paper.

\section{BACKGROUND AND PROBLEM DESCRIPTION}

\subsection{Hyperspectral image processing for detection problems}

Consider the problem of detecting flowers in a garden where a mixture of flowers and various plants are present [5]. Figure 1 illustrates the results where detection based on hyperspectral image processing is compared to that of conventional image processing. As shown in Figure 1(a), the object is detected in conventional image processing with edge detection using RGB information. Since this image contains many fragmented detected edges, isolating the desired target image becomes a challenge [6]. On the other hand, edge detection can be carried out after the hyperspectral image processing. The result is shown in Figure 1(b) in which only the images of flowers are detected. Such detection is possible because every material has an essential spectral property [7]. In this paper, Figure 1(b) is the ground truth image for comparisons.

Hyperspectral processing involves three key stages. The first step is the calibration stage. The image data produced by a sensor is manipulated to minimize sensor nonuniformity. The sensor is also calibrated by using the initially measured samples to consider the environment of measurement $[4,8]$. Each image cube contains a number of bands of spectral contents. For example, the image cube representing the garden of flowers as shown in Figure 2 consists of 30 bands of spectral information. Each band represents the information corresponding to a specific frequency range. Thus, a library (or spectral information) is constituted by a set of values, where the number of values corresponds to the number of

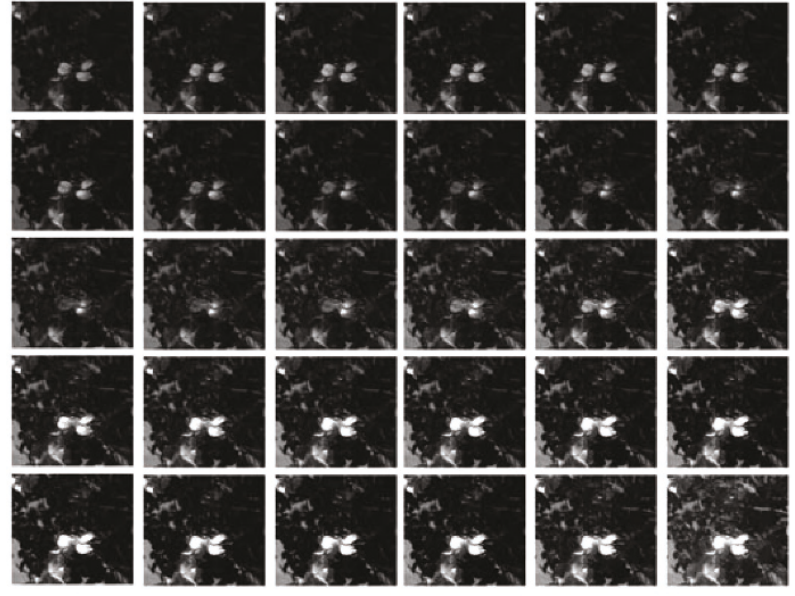

FIGURE 2: Illustration of images corresponding to different bands of the hyperspectral cube.

bands. In other words, every pixel in the cube is represented by a set of values; thus, a target (i.e., object image to be detected) is represented by numerous sets of values in a library. The second step is the detection stage. In the detection stage, target images are detected via isolating the portion of data which is highly correlated with the given target library. The target library contains spectral information about the object intended to be detected. The objective of the detection stage is to find out the image from the input cube that correlates with the spectral information stored in the target library. The third step is the visualization stage which collects detected image pixels and visualizes through color composition [8].

In this paper, we focus our discussion on the detection stage. Figure 3 illustrates the block diagram of hyperspectral processing. The main challenge of general hyperspectral image processing is the backside of its advantages: high volume and complexity of hyperspectral data. The performance of detection depends on the quality of spectral information stored in the target library. The main operation in the hyperspectral processing for target detection is to compare the input cube with the target library to determine correlation in terms of spectra. The detection is based on perceptual segmentation where spectra contents for each subband are correlated with the spectra contents stored in the library. However, not all bands are necessary since some may contain redundant information where they are compared to the target library. The easiest approach is to reduce the number of bands and the amount of library for processing. However, such reduction may eliminate the merit of hyperspectral processing. Hence, one of our objectives is to determine which bands are effective in detecting the target and selecting them accordingly. The effectiveness is measured in terms of the amount of target being detected with a fewer number of bands. In practice, a perfect target library, which is a set of all spectra comprising the target image, does not exist since objects exhibit different spectral characteristics which are sensitive to environmental factors such as lighting $[4,8,9]$. In the application of target detection, the basic library is a target 


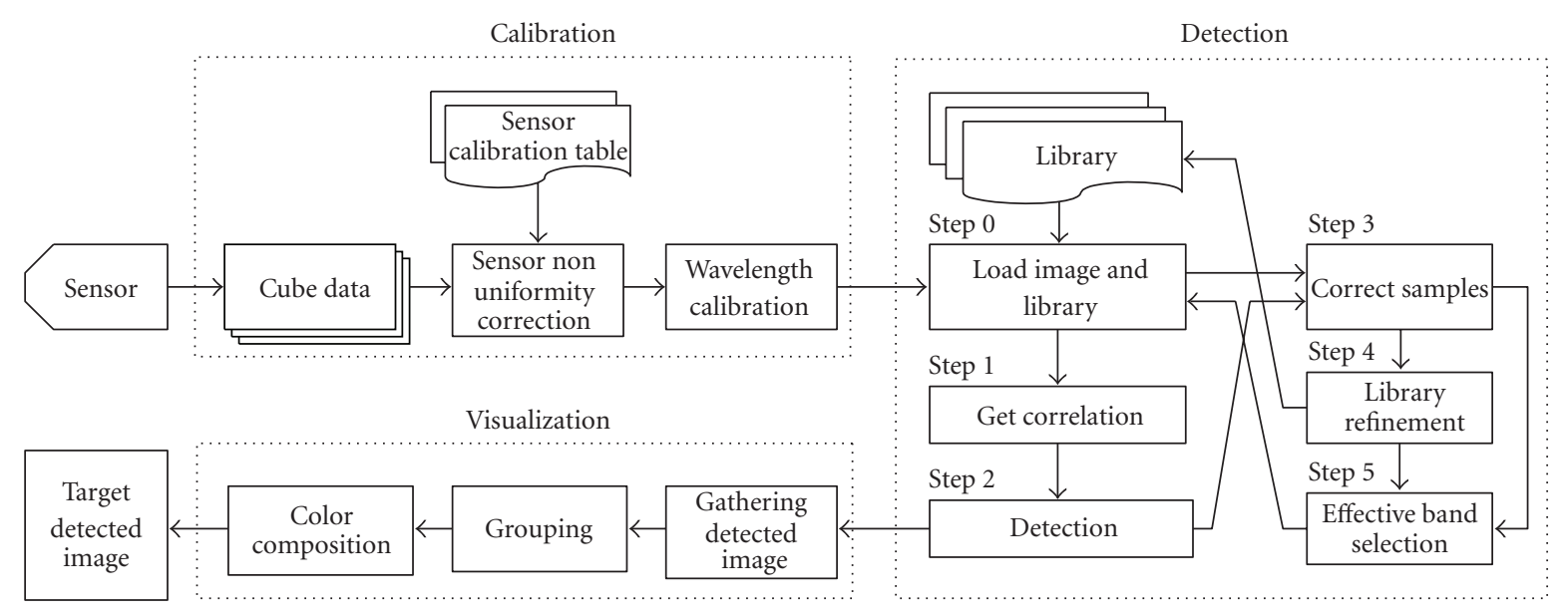

FIGURE 3: The block diagram of overall hyperspectral processing. A detailed description of steps is explained in Section 5.

spectrum which is generated in laboratories or measured in typical environments. Hence, the spectrum of the target image measured by different conditions results in mismatching the target library. Thus, we propose to refine the target library dynamically so that effective detection can be achieved with a small amount of target library information.

\subsection{Related work}

Traditional store-and-processing system performance is inadequate for real-time hyperspectral image processing without data reduction [3]. In this work, a fine-grain, lowmemory and single-instruction multiple-data (SIMD) processor is presented as an efficient computational solution for hyperspectral processing. However, the SIMD processor does not fully solve the higher resolution and a large number of band problems.

To minimize the volume of hyperspectral image processing, several data compression algorithms are proposed [10]. They achieve impressive compression ratios but could lose valuable information for detection or classification even though the error can be minimized by the clever compression algorithm. However, overall process is affected by the decompression complexity [11]. Statistical approach based on pattern recognition is one of the solutions for high dimensionality of hyperspectral image processing. It uses a small number of reference measurements to distinguish material identification. However, it requires a large number of sample pixels to determine accurate probability density function [11].

Even though hyperspectral image processing uses hundreds of bands to detect or classify targets, there is redundancy which means that partial bands efficiently accomplish the edge detection as described in $[11,12]$. In [11], the band selection is based on the band add-on (BAO) procedure that chooses an initial pair of bands and classifies two spectra by correlation, and then adds additional bands that increase the correlation of two spectra. It is a feasible solution to determine effective bands when an unknown pixel is classified by using many reference classes. A set of best-bases feature ex- traction algorithms is proposed for classification of hyperspectral data as well [13]. This method is simple, fast, and highly effective so that it can reduce the input space from 183 dimensions to less than four dimensions in many cases. However, this approach is based on classification so that it is suitable when a spectrum of a pixel is classified by many numbers of libraries. In the application domain of target detection, the input image is compared to a few libraries which represent the spectrum contents of the target.

\subsection{Correlation coefficient of image $(A)$}

Correlation coefficient, $A$, is a measure of similarity between the stored spectra in a target library and the obtained spectra from sensors. The high value of correlation indicates the high degree of similarity between two spectra [14]. The correlation coefficient is defined as

$$
A=1-\cos ^{-1}\left(\frac{\sum_{i=1}^{N_{T}} t_{i} r_{i}}{\sqrt{\sum_{i=1}^{N_{T}} t_{i}^{2}} \sqrt{\sum_{i=1}^{N_{T}} r_{i}^{2}}}\right),
$$

where $N_{T}$ is the number of bands in input spectrum, $t_{i}$ is the test spectrum of the $i$ th band, and $r_{i}$ is the reference spectrum of the $i$ th band. The value of correlation defines a degree of similarity between input spectrum and target spectrum stored in the target library.

The input spectra of an object is compared to the spectra in the target library. This comparison is based on the correlation coefficient. In this paper, we define $A_{t}$ as the minimum correlation coefficient value which recognizes the target between unknown spectra. When the correlation value is higher than or equal to $A_{t}$, the object is assumed to be matched with the data in the target library. Thus, the value is used as an indicator for the degree of confidence in detection.

If we use lower $A_{t}$ to detect targets, it increases the possibility of wrong detection which means that some backgrounds are detected as a target. However, if the numbers of 


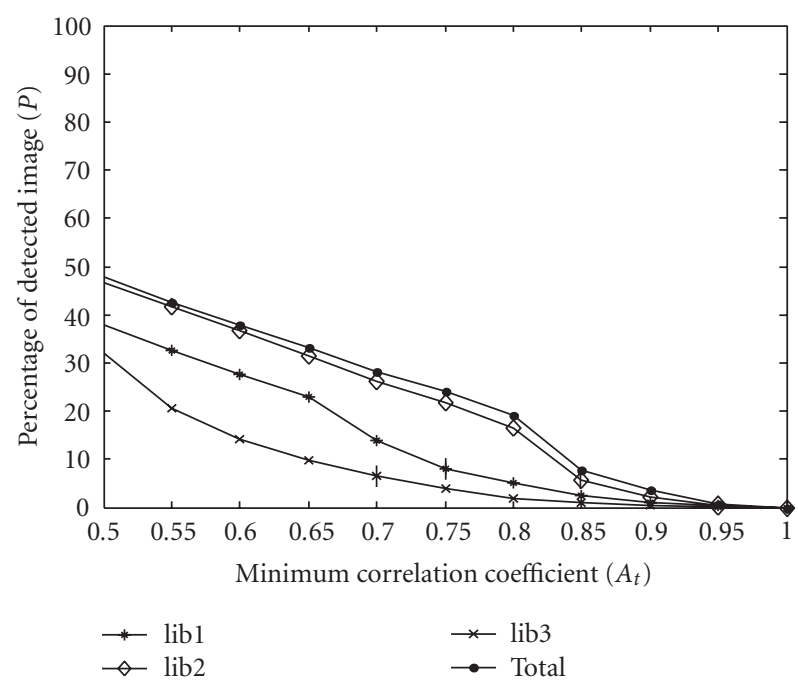

Figure 4: Relationship between the correlation value used and detected image percentage of detected image $(P)$. Thirty one bands of input image data are used in the simulation.

libraries and bands applied in detection is increased, the performance of target detection is improved. However, even if all possible information is used to detect targets, there is a limit value where target and background cannot be isolated. Thus, the minimum correlation coefficient $\left(A_{t}\right)$ is related to the similarity within the target and background. We define $A_{b}$ as a maximum correlation value where any correlation value below $A_{b}$ is considered to be a background, which means that the pixel is not a target at least. The detected image with the correlation value below $A_{b}$ may not be the interest of objects which may capture a large portion of the background.

\subsection{Percentage of detected image $(P)$}

Percentage of detected image $(P)$ shows the effectiveness of selected bands in the detection process. Figure 4 illustrates the relationship between the correlation coefficients and percentage of detected image $(P)$ where three types of target libraries are used. When the given correlation coefficient $A_{t}$ is 1 , the value of percentage of detected image $(P)$ is very low (i.e., approaches zero). For all libraries, when the correlation coefficient is increased, the percentage of detected image $(P)$ is decreased. We define $A_{t}$ as the correlation value where the change in percentage of detected image $(P)$ is smaller than some value $\delta$ as we increase the value of the correlation coefficient.

Figure 5 shows the simulation results of the detected image as a function of the minimum correlation values for one target library, lib1. The detected images are shown for different minimum correlation values: $0.70,0.75$, and 0.85 . In the case where $A_{t}$ of lib1 is 0.7 , unwanted objects that satisfy the minimum correlation value are detected as a target. However, as $A_{t}$ is increased to 0.85 , the unwanted objects almost disappear in the detection at the cost of losing the target image. At

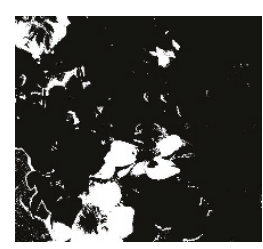

(a) $A_{t}=0.7$

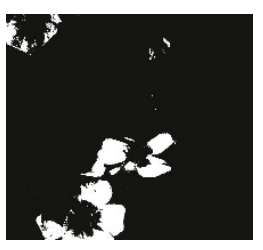

(b) $A_{t}=0.75$

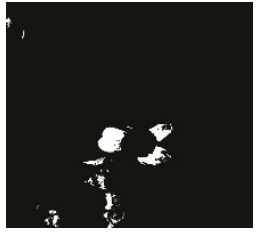

(c) $A_{t}=0.85$
FIGURE 5: The result of detected image as a function of correlation values $A_{t}$ for lib1. Thirty one input bands are used and processed with one library.

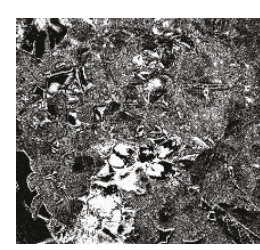

(a) 2 bands

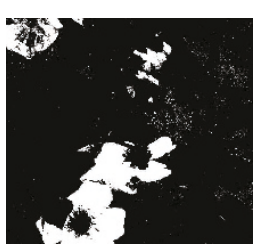

(b) 4 bands

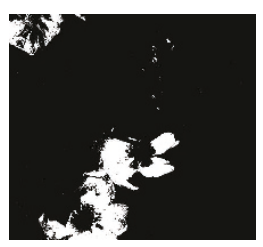

(c) 16 bands
FIGURE 6: The results of detected image as a function of the number of bands used out of 31 input bands.

the minimum correlation $A_{t}$ of 0.85 , the process tries to find only the image from the input that is highly correlated with the target library.

The values of percentage of detected image $(P)$ have two interpretations. First, the higher value of percentage of detected image $(P)$ (i.e., more images have been detected) implies that more target images are detected. Second, the higher value of percentage of detected image $(P)$ can imply that some of the detected images are not the target. Hence, detection depends on the number of libraries (spectral information) and their qualities as well as the minimum correlation values used in the process.

Under the assumption which multiple libraries are used in the detection, we define the total percentage of detected image $\left(P_{T}\right)$ as follows:

$$
P_{T}=\sum_{l} P\left(l, A_{t}\right)
$$

where $l$ is the index of each library and $P\left(l, A_{t}\right)$ is the percentage of detected image $(P)$ value at the correlation value $A_{t}$ when library $l$ is used. We will use the total percentage of detected image $(P)$ as an indicator for detection performance.

\section{TARGET DETECTION}

\subsection{Effects of number of bands}

Since the motivation of our work is to use the smaller number of bands for detecting the target, we investigate the effects of the number of bands on detection performance. Thus, the goal is to minimize the total percentage of detected image $\left(P_{T}\right)$ at the minimum correlation $\left(A_{t}\right)$ given the number of bands $\left(N_{E}\right)$. 


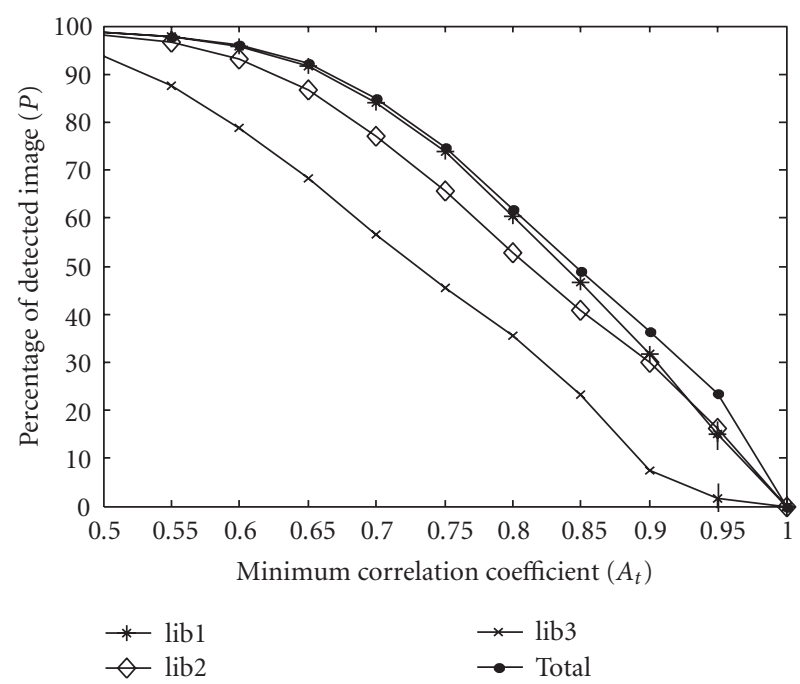

Figure 7: Relationship between the correlation values and percentage of detected image $(P)$ when clustered bands $(27,28,29,30)$ are used in the detection.

Figure 6 shows the detected image where a partial number of bands are used to detect flowers. When the number of bands, $N_{E}$, is equal to 2 , the detected image includes the target image as well as other unwanted background images. It implies that two bands are not effectively isolating the target image. When the number of bands is more than 4 , the detected images become isolated and percentage of detected image $(P)$ is lower than that of the image generated with 2 bands. However, there is only slight improvement (the total percentage of detected image $(P)$ is decreased) from 4 bands to 16 bands.

We define the degree of effectiveness in terms of the total percentage of detected image $\left(P_{T}\right)$. As shown in Figure 6(a), total percentage of detected image $\left(P_{T}\right)$ is higher than that shown in Figures 6(b) and 6(c) (i.e., more images are shown). However, total percentage of detected image $\left(P_{T}\right)$ is improved (reduced) very slightly from 4 bands to 16 bands. This shows that the complete use of the bands is not always necessary for detecting the target from the input image.

\subsection{Redundancy between bands}

To use the partial number of bands, the simplest approach is to select bands in random. In this section, we consider two types of band selection in order to characterize the effect of band selection on detection performance. We investigate the redundancy within the bands.

\subsubsection{Clustered bands}

Cluster band selection selects $N_{E}$ consecutive bands. Figure 7 shows the relationship between the correlation coefficient and percentage of detected image $(P)$ when 4 consecutive bands are selected out of 31 possible bands. The selected

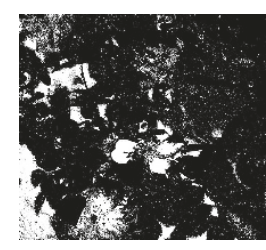

(a) With lib1

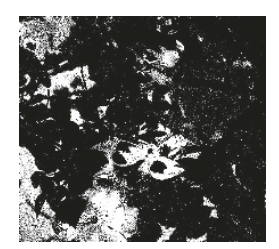

(b) With lib2

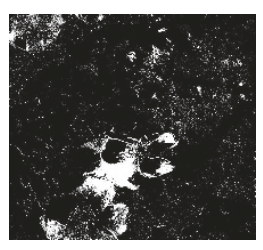

(c) With lib3

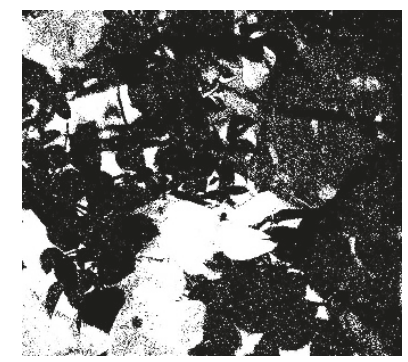

(d) Detection with clusters

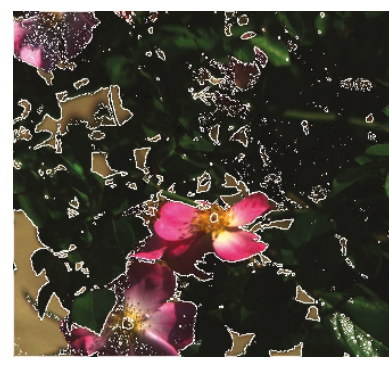

(e) Detected image with full colors
FIGURE 8: Result of detected image when clustered bands are used in the detection. Bands used are $(27,28,29,30)$.

bands are $(27,28,29,30)$. The figure shows a much higher percentage of detected image $(P)$ for the entire range of correlation values when it is compared to that of Figure 4. Thus, the figure indicates that it has detected more image from the background. In this situation, it is likely that the detected image contains a lot of unwanted images.

The analysis with the percentage of detected image $(P)$ is proven by the detected image illustrated in Figure 8 . Each of the three libraries were not effective in detecting the flowers. Even with the correlation coefficient of 0.95 , the target is not separated from the background. This simulation suggested that those clustered bands contain redundancy and the clustered bands are not effective in detecting the target. Similar results were obtained when the other sets of clusters are used. Thus, the clustering is not an effective way to select the bands for detection.

\subsubsection{Maximum separation bands}

On the other hand, we select the bands that are maximally separated. There are several combinations of sets of bands. Figure 9 shows the relationship between correlation and percentage of detected image $(P)$ where bands are selected by maximal separation as $(2,10,18,26)$.

As shown in Figure 9, percentage of detected image $(P)$ values of each library as well as the total percentage of detected image $\left(P_{T}\right)$ are much lower than that for the entire range of the correlation values. For example, the total percentage of detected image $\left(P_{T}\right)$ of clustering case at $A_{t}=80$ is 70 while maximum separation case at $A_{t}=80$ is 40 . This implies that the maximal separation performs better than the clustering at any minimum correlation value. The detected image by each library shown in Figure 10 contains only the flowers. This is improved detection much over the clustering 


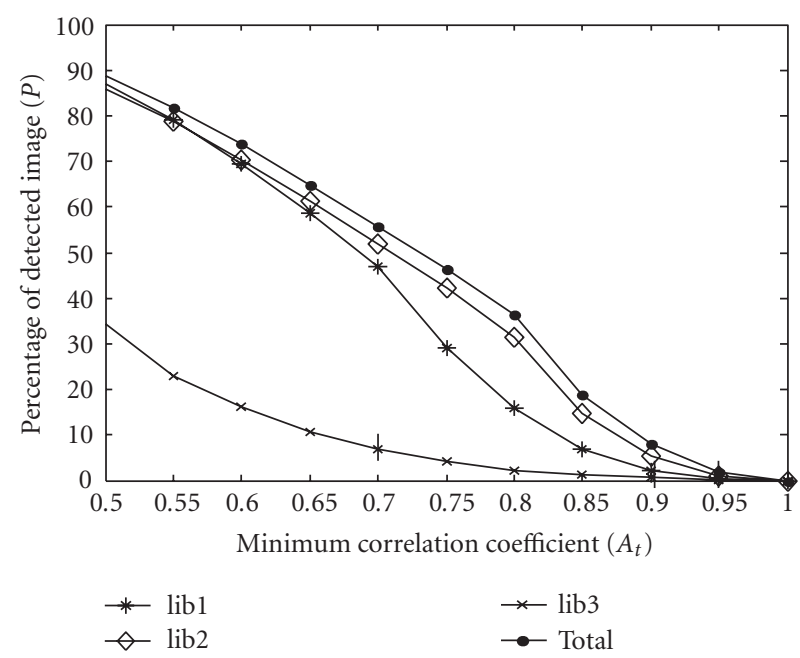

FIGURE 9: Relationship between the correlation values and percentage of detected image $(P)$ when maximum separation bands are used in the detection. Band used are $(2,10,18,26)$.

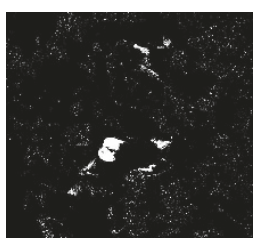

(a) With lib1

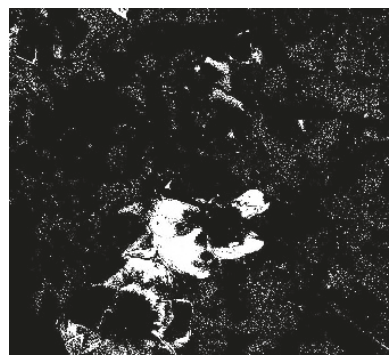

(d) Detection with maximum separation

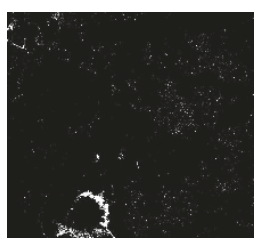

(b) With lib3

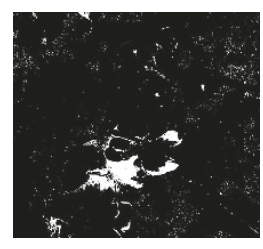

(c) With lib3
FIGURE 10: Result of detected image when maximum separation bands are used in the detection. Bands used are $(2,10,18,26)$.

method. Figure 10(d) illustrates the detected image when all three libraries are used.

However, in the results generated by the maximum separation, some of the targets were lost. Similar results are obtained with a different set of bands $(4,12,20,28)$. The detected images by three target libraries are illustrated in Figure 11 . The band set $(4,12,20,28)$ performs better than the band set $(2,10,18,26)$ in detecting and isolating the target images. This implies that while the maximum separation scheme is better than the clustering, more bands may be necessary since the total percentage of detected image $\left(P_{T}\right)$ value obtained is much higher than the case of 31 bands. We will present an effective band selection scheme in Section 4.

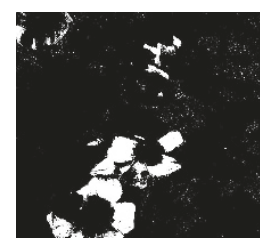

(a) With lib1

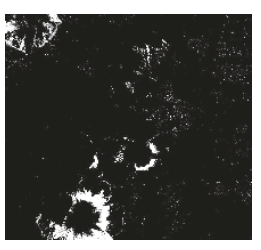

(b) With lib2

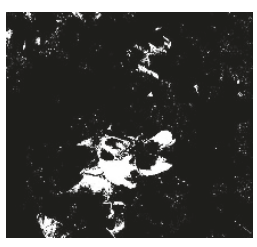

(c) With lib3
FIGURE 11: Result of detected image when maximum separation bands are used in the detection. Bands used are $(4,12,20,28)$.

\subsubsection{Observation}

We can observe from the results that detected images are improved when the percentage of detected image $(P)$ value is low for the given correlation values. This observation coincides when we compare Figures 4, 7, and 9. Percentage of detected image $(P)$ is the lowest when all bands are used for given correlation value. We will consider an approach for selecting bands in the next section.

When the number of bands is increased, percentage of detected image $(P)$ is reduced and then it is saturated. This means that a target can be detected by using only partial bands because some bands have enough information to detect a target.

\section{COMPLEXITY REDUCTION STRATEGY}

The main objective in reducing computational complexity is to determine the minimum number of bands used in the detection process as well as selecting a specific set of bands. In this section, we first define the band contribution coefficient and present a band selection strategy based on the coefficient.

\subsection{Band contribution in detection}

Library usually has several spectra for a target because the spectrum depends on the measurement part of the target and the condition of light sources. Figure 12 is an example of spectra for library and background, which shows three libraries and two background spectra. When the spectral information of the target is highly different from the background, the target detection is easier. In Figure 12, the spectrum of lib1 from the 18th band to the 31 st band is saturated. Also, spectrum waveform of lib2 is similar to lib3. However, the magnitude is different within the two libraries, background 1 is extracted from leaves and background 2 is from the back of a scene.

The effectiveness of the $k$ th band of the $l$ th library, $e_{l, k}$, is defined as

$$
e_{l, k}=\frac{\left|\sum_{b=1}^{N_{B}}\left(l_{l, k}-b_{b, k}\right)\right|}{N_{B}}
$$

where $N_{B}$ is the number of backgrounds, $l_{l, k}$ is the $k$ th spectrum content in the $l$ th library, and $b_{b, k}$ is the $k$ th spectrum content in the $b$ th background. 


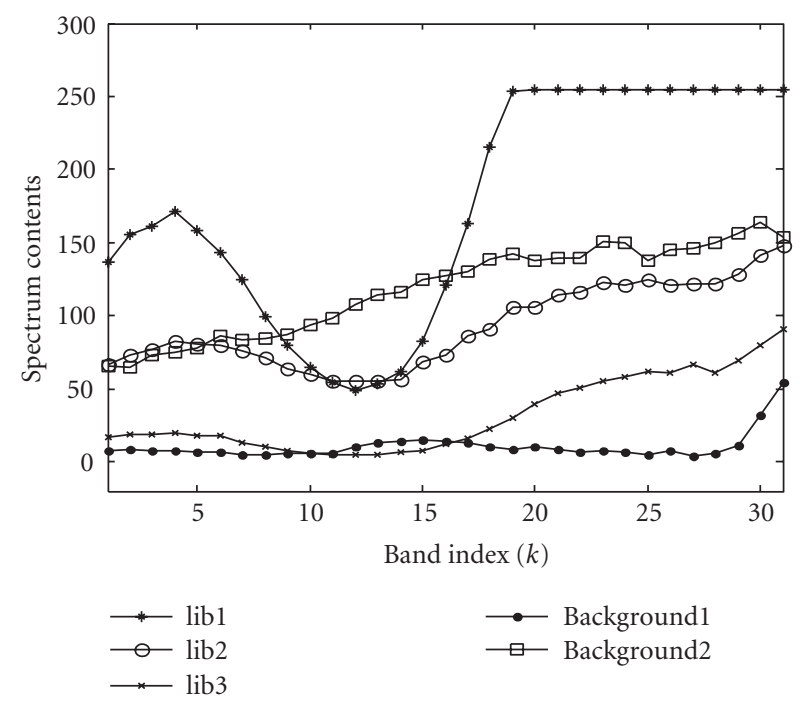

FIGURE 12: The comparison between spectrum of target libraries and the spectrum of the background of input bands.

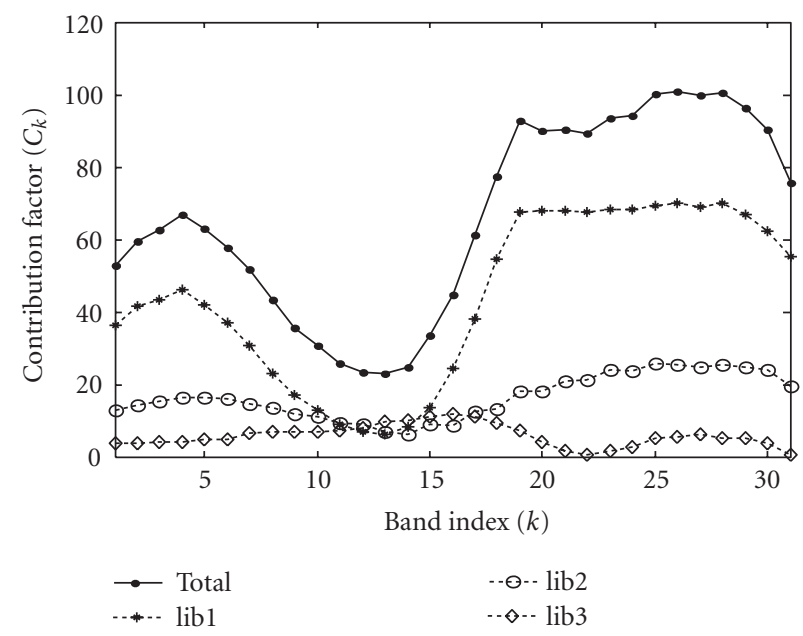

FIGURE 13: Illustration of contribution coefficient of each band.

If a spectrum of a target is similar to that of data in the library, target detection is achieved more effectively; we will define the effectiveness as contribution. The contribution coefficient $(c)$ is defined as

$$
c_{k}=\frac{\sum_{l=1}^{N_{\mathrm{lib}}} e_{l, k}}{N_{\mathrm{lib}}}
$$

where $c_{k}$ is the contribution of the $k$ th band and $N_{\text {lib }}$ is the number of libraries.

The relationship between the contribution factor and the number of bands is illustrated in Figure 13. Contribution of lib2 and lib3 is less than 20 while lib1 has much higher contribution than other two libraries. Thus, the contribution of lib1 is dominant as shown in Figure 13.
Even though the contribution coefficient is not an absolute indicator for detection, the coefficient is considered to be one of the factors for isolating the target. To obtain the contribution, we need to choose samples of backgrounds. Samples are randomly selected in a scene, and then each sample is verified to be a background or an applicant of a target by using the maximum correlation coefficient $\left(A_{b}\right)$. If the correlation coefficients between an input spectrum and all of the libraries are lower than $A_{b}$, the input spectrum is considered a background. Also, $A_{b}$ is experimentally decided depending on an application. Although background and library can be highly correlated, the contribution factor is a powerful factor under the condition of which $A_{b}$ is lower than $A_{t}$.

\subsection{Effective band selection}

Since the contribution coefficient represents the effectiveness to detect targets, it has a benefit for effective band selection. However, if the high contribution bands are selected, it may lead to select clustered bands (i.e., bands 27, 28, 29, 30).

From the definition of correlation in (1), the correlation of library and background is basically the variation of the difference in two spectra. For example, if the spectrum contents in a reference are $(10,20,40,60,50,30)$ and the test spectrum has 10 times higher value of contents like $(100,200,400,600,500,300)$, the correlation between two spectra is 1 , which means that two spectra are perfectly correlated since the variations of spectrum contents between adjacent bands are the same.

Thus, effective bands represent the variation of differences between the library and the background. Since contribution is related to the difference between the library and the background, isolating the target and background in lower $A_{t}$ can be one of the solutions in maximally separated bands. To maximally separate the contribution of selected bands, the first band has minimum contribution and the last band has maximum contribution. The contribution of the $k$ th bands is $((\max C)-(\min C)) /\left(N_{E}-1\right) \times k+(\min C)$, where $(\max C)$ and $(\min C)$ are the values of maximum and minimum contributions, respectively.

For example, let us assume a series of contributions is $(90,180,360,540,450,270)$. Since the contribution of the 1 st band is minimum and the 4th band is maximum, the 1st and the 4 th are selected. Then, since the gap of selected bands is $150(=(540-90) / 3)$, contributions of second and third bands are approximately 240 and 390, respectively. Since the contribution values of the 6th and the 3rd bands are close to 240 and 390 , the 6th and the 3 rd bands are selected as effective bands. Figure 14 shows the result of target detection when effective bands are selected. The result is similar to the one in the case where full bands are used.

\subsection{Library selection}

We have observed that some target libraries work better in detecting the target than other target libraries. Theoretically, a larger set of target libraries will enhance the detection but at the cost of computational complexity. We investigate the target library selection in cases where the finite number of 


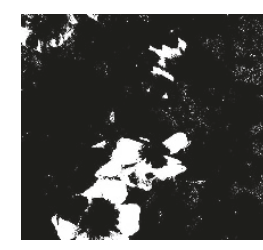

(a) With lib1

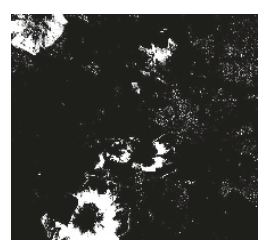

(b) With lib2

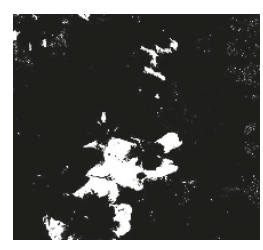

(c) With lib3

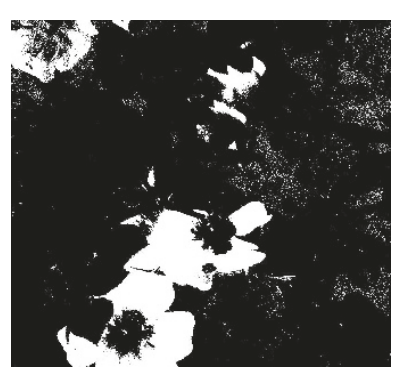

(d) Detection with effective band selection

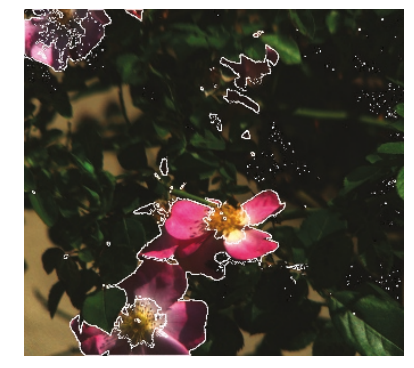

(e) Detected image with full colors
Figure 14: Result of detected image when effective band selection strategy is used in the detection.

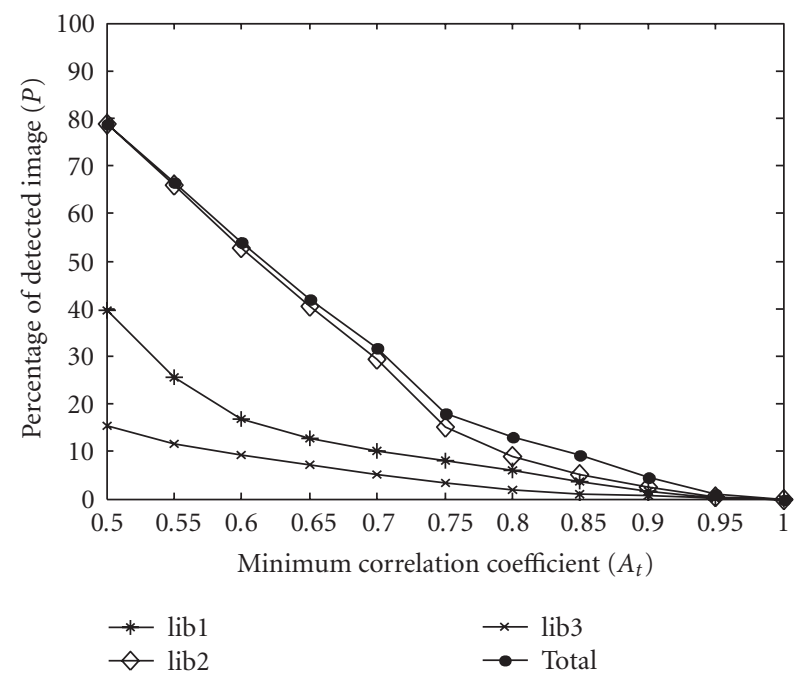

FIGURE 15: Relationship between the correlation values and percentage of detected image $(P)$ when effective band selection strategy is used.

target libraries is to be used for reducing the computational complexity. However, the best possible sets of target libraries cannot be generated or obtained before the processing. However, the target library can be improved during the detection process.

In Figure 15, the total percentage of detected image $\left(P_{T}\right)$ from lib1, lib2, and lib3 is $14 \%$ when $A_{t}$ is equal to 0.8 . Even though lib1 is more effective to detect targets than other libraries, lib2 or lib3 can detect the different part of the targets.

Note that the lower value of $P_{T}$ does not imply that the performance is better. It merely suggests that there is a high

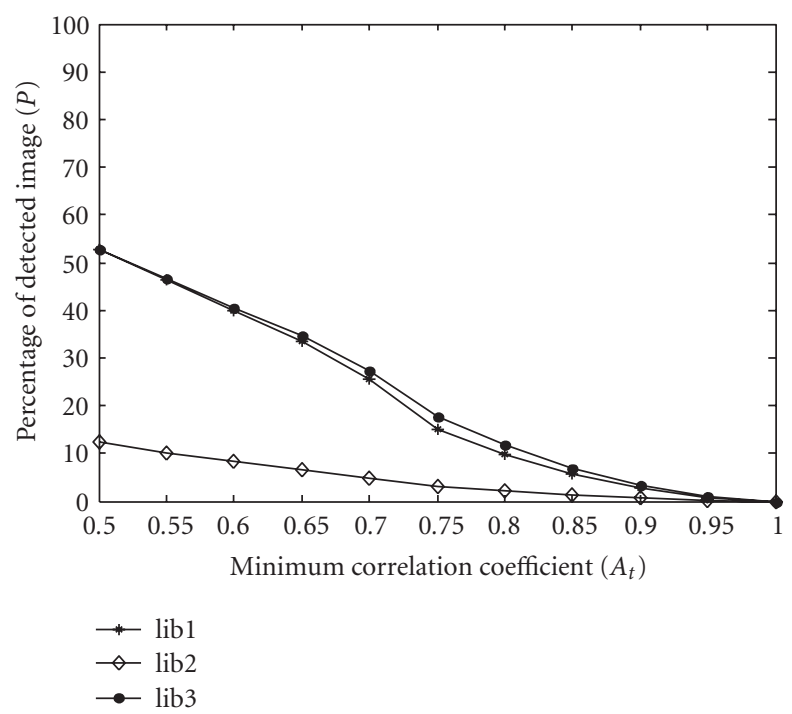

FIGURE 16: Relationship between the correlation values and the percentage of detected image $(P)$ when two libraries are used.

probability that the detected image is only a target. Figure 16 shows the relationship between percentage of detected image $(P)$ and correlation coefficient when it has two libraries (lib2 and lib3). In addition, when several libraries are used, more effective libraries will produce bigger contributions.

Figure 17 shows the result of target detection where lib2 and lib3 are used. Figures $17(\mathrm{a})$ and 17(b) have 5.71\% and $4.71 \%$ of percentage of detected image $(P)$, respectively. Since the total percentage of detected image $\left(P_{T}\right)$ is $10.39 \%$, two detected areas are slightly overlapped.

\subsection{Library refinement}

One important aspect that we have discussed in this paper is that the performance depends on the quality of the target library. Library refinement improves the detection process. The overall process starts with a set of basic libraries. Once a target image is detected, the target library from the detected image is refined. The refined library has all spectrums of the detected target. Once the refined library is generated, the library is applied in lieu of the basic library.

Figure 18 shows the results of library refinement where the detected image has 0.9 of the correlation coefficient. Figure 18(a) uses the basic library and Figures 18(b) and 18 (c) use the refined library. Since $A_{t}$ is not 1 (perfect correlation value), a background image is detected as a target. Hence, the chosen target image with library refinement is a candidate of the new library. The randomly selected target image is compared to the basic library each time. If the correlation between the new library candidate and basic library satisfies the condition $\left(\geq A_{t}\right)$, the current library is replaced by the new library candidate. Otherwise, the basic library is used in the process.

In Figure 19, refined libraries are shown by the dashed line where all refined libraries satisfy the condition of correlation $\left(A_{t}=0.9\right)$. The refined library can be adopted in a variety of light source conditions. 


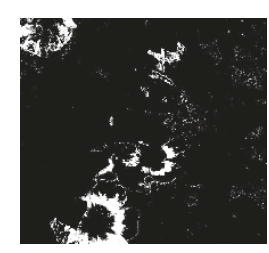

(a) With lib1 (5.71\%)

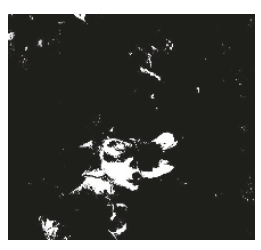

(b) With lib2 (4.71\%)

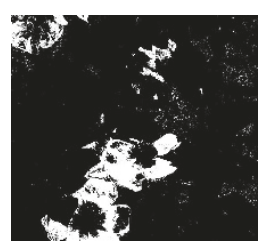

(c) With lib1 and lib2 $(10.39 \%)$

FIGURE 17: Library selection.

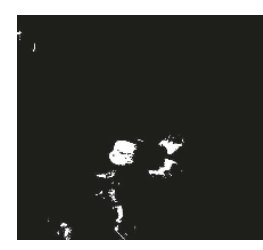

(a) Basic library

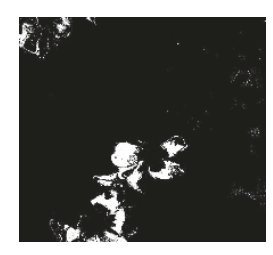

(b) Case 1 of refined library

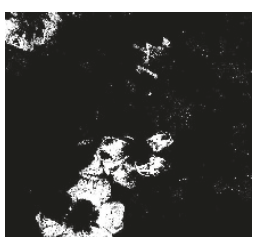

(c) Case 2 of refined library

FIGURE 18: Result of detected images when the libraries are refined from detected samples $\left(A_{t}=0.9\right)$.

\section{ALGORITHM DESIGN}

\subsection{Algorithm overview}

Figure 20 illustrates the overall algorithm for detecting and isolating target images in processing where the algorithm has two processing flows. The right-hand side is for comparing the input cube with the target libraries. The left-hand side has two parts where the target library is refined and the effective band selection is performed.

We assume that the basic parameters are loaded in Step 1. The basic parameters are the number of bands $\left(N_{E}\right)$, the number of libraries $\left(N_{\text {lib }}\right)$, the number of background samples $\left(N_{B}\right)$ and the number of target samples $\left(N_{T}\right)$, the minimum correlation coefficient between library and target $\left(A_{t}\right)$, and the maximum correlation coefficient between library and background $\left(A_{b}\right)$. The basic parameters are based on the type of the target and detecting environment. The output of processing is a series of end members which represents a type of a target.

\subsection{Iteration process}

The algorithm repeats the following steps until $i=N_{x}$ and $j=N_{y}$ for a cube.

Step 1. Load spectrum contents in a pixel $(i, j)$ and libraries. Initially, maximally separated bands are selected as effective bands. Then, from the next cube, effective bands are selected by Step 6 . Thus, the number of spectrum contents is the same as the number of effective bands $\left(N_{E}\right)$.

Step 2. Compute the correlation coefficient between an input spectrum and the $l$ th library.
Step 3. Classify each pixel $(i, j)$ whether it is a target or a background; Step 3.1 is for target detection, and Step 3.2 is for background detection.

Step 3.1. If the correlation coefficient $(A)$ is higher than $A_{t}$, it is considered to be a target. Even though the libraries are only for a target, the detected results are saved separately for library refinement.

Step 3.2. If $A$ is lower than $A_{b}$, it can be a candidate for the background. Even if a spectrum of a pixel is not considered to be a target, it can be a target of other libraries so that there is a tag bit which takes either false (0) or true value (1). After the loop for library refinement is completed with tag bit 1 , it is classified as a background.

If the value $A$ is between $A_{b}$ and $A_{t}$, it is impossible for the pixel to be classified due to insufficient information. Thus, to save end members, $N_{x} \times N_{y} \times\left(N_{\text {lib }}+1\right)$ size of bit memories is required since the area size of $x-y$ plane is $N_{x} \times N_{y}$ and each end member requires a bit memory to save the information where 1 is the end member and 0 is the unknown object. In addition, since the number of bits to save the type of the end members in a pixel is the sum of the number of libraries $\left(N_{\text {lib }}\right)$ and a background, the $\left(N_{\text {lib }}+1\right)$ bits are required for end members. For example, if there are three libraries, the required end member bits are 4 bits. Furthermore, if all end member bits are 0 (where background bit is also 0 ), it is classified as a background.

Step 4. Choose samples for background and target. To represent the spectrum of the background area, the samples of background are randomly selected where the number of background samples is $N_{B}$. For library refinement, each library uses one sample as a candidate to replace the current 


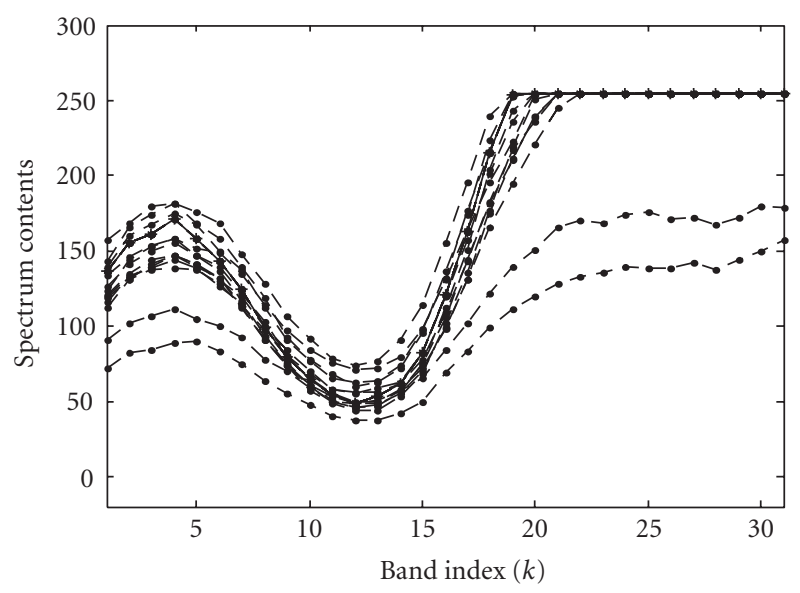

(a) With lib1

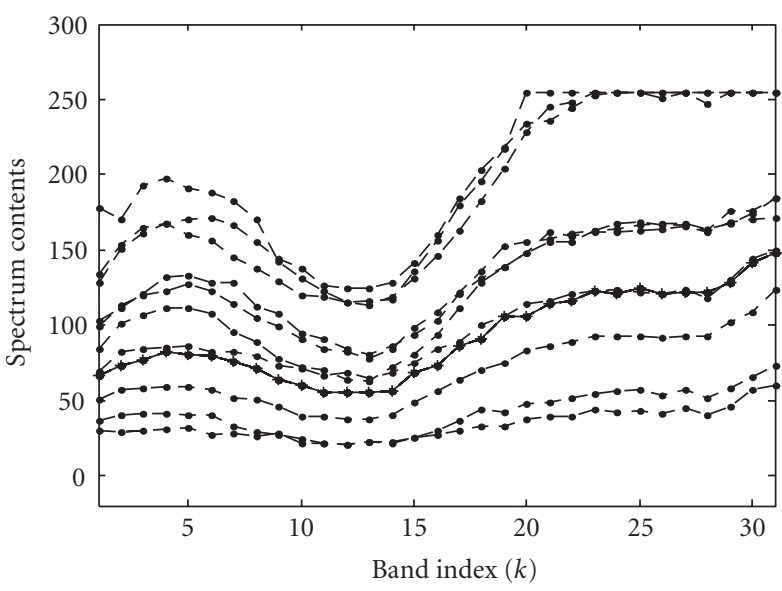

(b) With lib2

FIgURE 19: The refined libraries of lib1 and lib2 $\left(A_{t}=0.9\right)$.
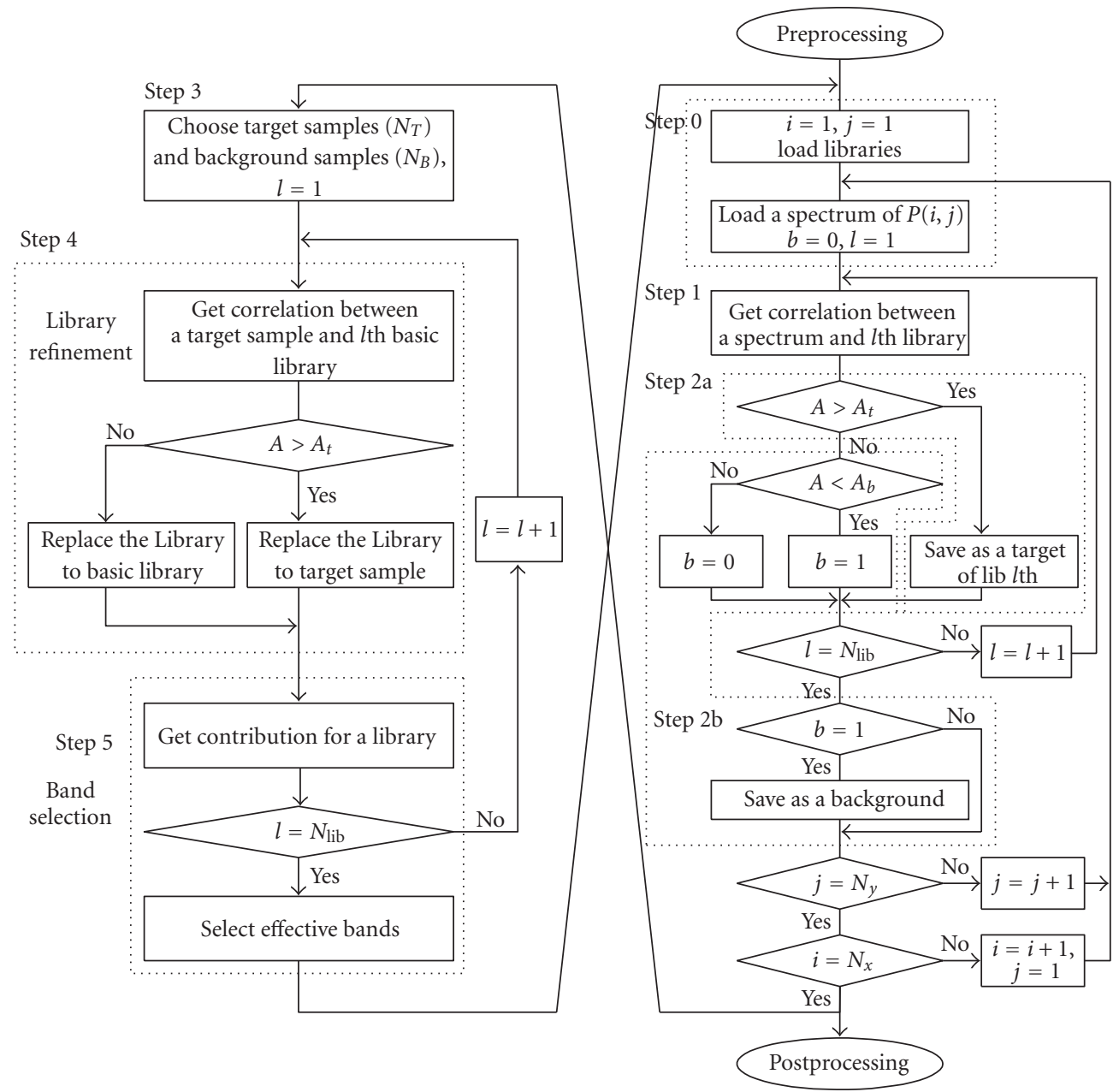

FIgURE 20: Flowchart of proposed algorithm for the detection process. 


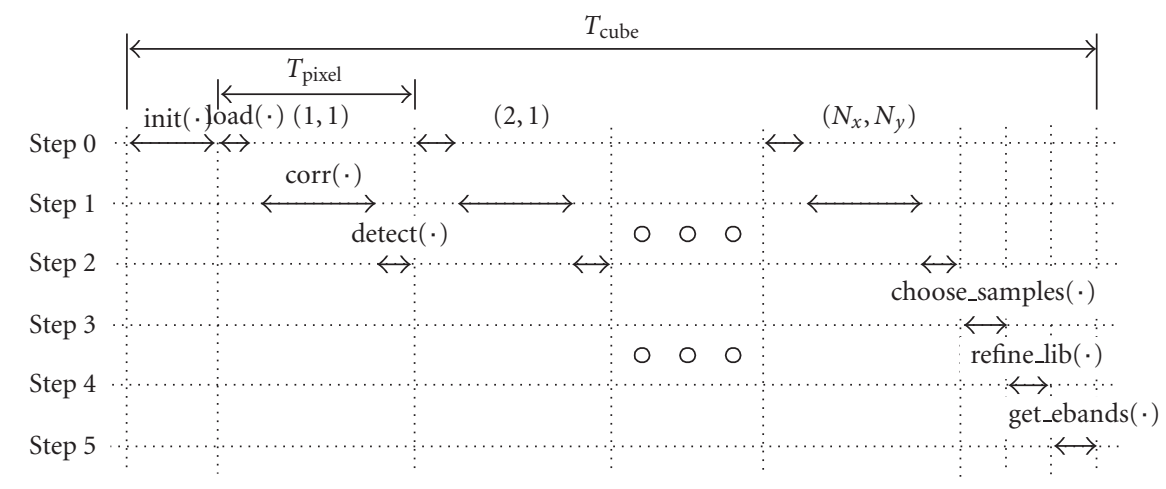

Figure 21: Time flow in processing.

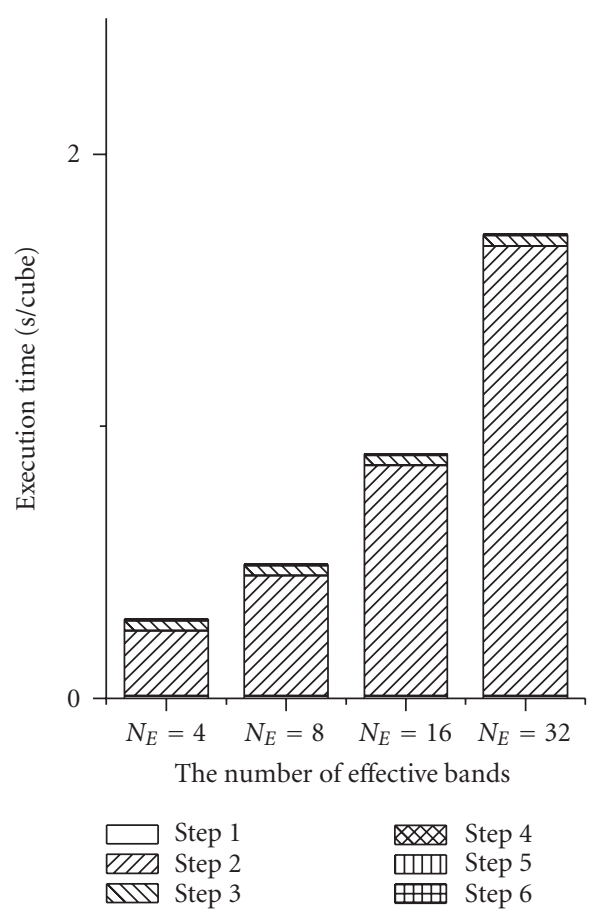

(a) $N_{E}$

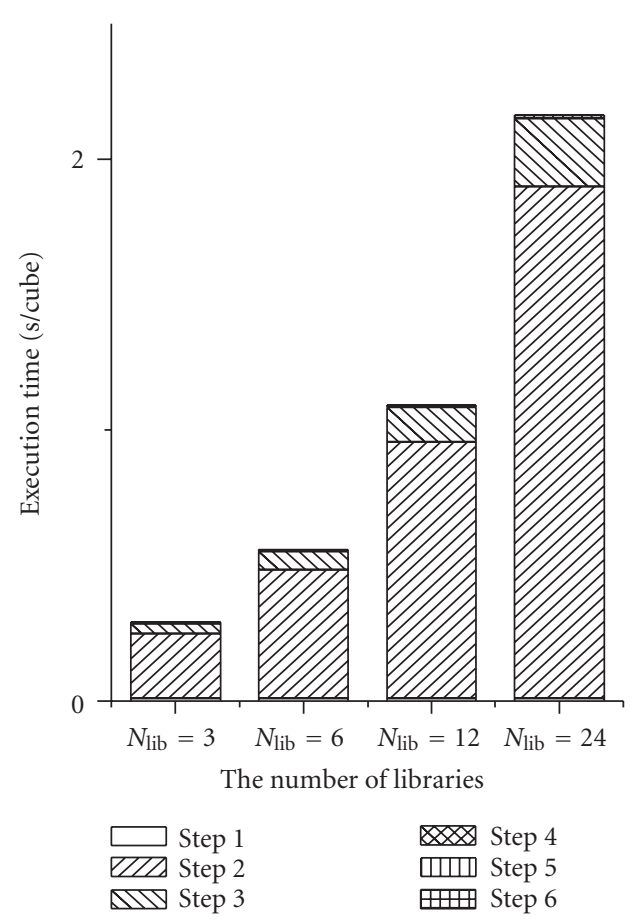

(b) $N_{\text {lib }}$

FIgURe 22: The execution time in function of number of effective bands and the number of libraries, where (a) $N_{\text {lib }}=3, N_{x}=820, N_{y}=748$, $N_{B}=1000, N_{T}=1000 ;$ (b) $N_{E}=4, N_{x}=820, N_{y}=748, N_{B}=1000, N_{T}=1000$.

library. We assume the area of targets is much smaller than the area of background. All of the detected targets are counted and randomly selected in endmembers. If we count all backgrounds to select randomly, they make excessive data loading so that we select $N_{B}$ random pixels from the entire image.

Step 5. Refine current library. The sample is a candidate for the new library. Since the partial number of bands is used to obtain correlation in Steps 2 and 3, the sample is compared to the basic library again for entire bands. If $A$ is higher than $A_{t}$ where the correlation between the $l$ th library and a spectrum of a sample uses all of the bands of which size is $N_{z}$, the candidate replaces the current library. Otherwise, the current library goes back to the basic library. The refined library is saved to a memory for libraries.

Step 6. Select effective bands. From Step 5, we obtained the new library so that effective bands are changed to support the new library. Since the band selection is based on contribution, $\left(N_{\text {lib }} \times N_{B}\right)$ operations are required to get contribution (c). From the distribution of contribution coefficient, $N_{E}$ bands are selected.

Figure 21 shows the timing flow of hyperspectral processing algorithm. $T_{\text {init }}$ represents the time interval for loading libraries and several coefficients such as the minimum correlation coefficient between library and input image $\left(A_{t}\right)$, the 


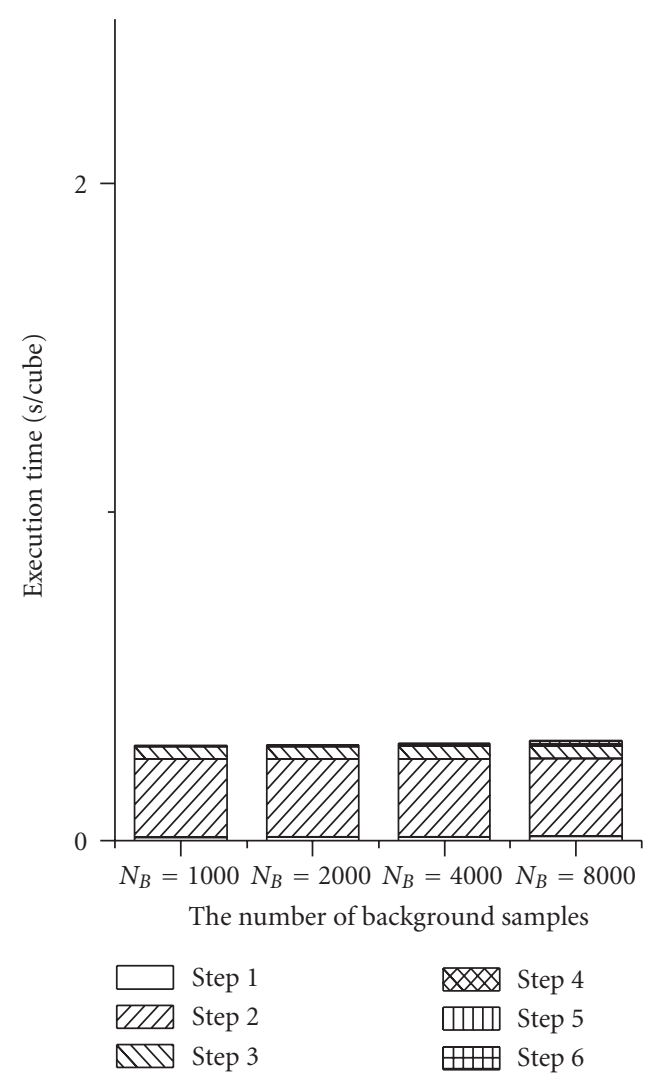

(a) $N_{B}$

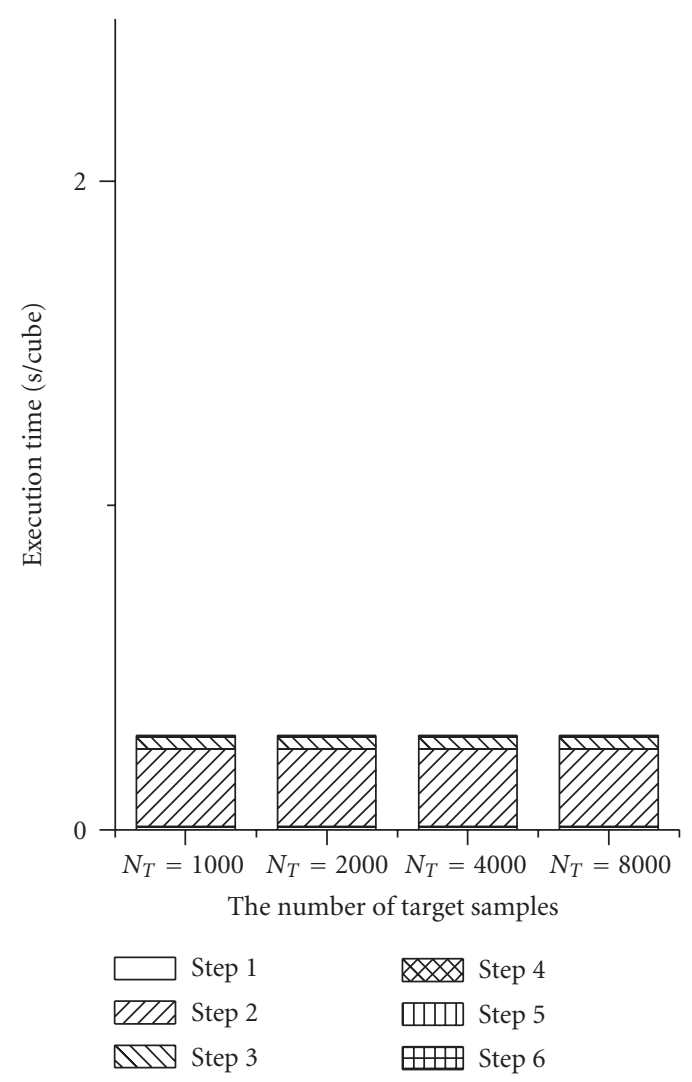

(b) $N_{T}$

FIgURE 23: The execution time in function of number of background samples or the number of target samples, where (a) $N_{E}=4, N_{\text {lib }}=3$, $N_{x}=820, N_{y}=748, N_{T}=1000$; (b) $N_{E}=4, N_{\text {lib }}=3, N_{x}=820, N_{y}=748, N_{B}=1000$.

maximum correlation coefficient between a library and an input image $\left(A_{b}\right)$, the number of libraries $\left(N_{\mathrm{lib}}\right)$, the number of target samples $\left(N_{T}\right)$, the number of background samples $\left(N_{B}\right)$, and the number of effective bands $\left(N_{E}\right) . T_{\text {pixel }}$ is the processing time for a pixel and the sum of $T_{\text {load }}, T_{\text {corr }}$ and $T_{\text {detect }}$ from Step 1 to Step 3, where $T_{\text {load }}$ is the required time for the function load( $\cdot)$ in Step $1, T_{\text {corr }}$ is for the function $\operatorname{corr}(\cdot)$ in Step 2, and $T_{\text {detect }}$ is the required time for the function $\operatorname{detect}(\cdot)$ in Step 3. Thus, the total required time for a cube is $T_{\text {init }}+T_{\text {pixel }} \times N_{x} \times N_{y}+T_{\text {choose_samples }}+$ $T_{\text {refine_lib }}+T_{\text {get_ebands }}$, where $T_{\text {choose_samples }}$ is the required time for the function choose_samples( $\cdot)$ in Step $4, T_{\text {refine_lib }}$ is for the function of refine_lib( $\cdot)$ in Step 5, and $T_{\text {get_ebands(.) }}$ is the required time for the function get_ebands $(\cdot)$ in Step 6.

\subsection{Complexity}

The complexity of this algorithm has been estimated by TMS320C6713 $(300 \mathrm{MHz})$ based on the VLIW architecture. The internal program memory is structured so that a total of eight instructions can be fetched in every cycle $[15,16]$. We estimate the execution time from the instruction cycle count using Code Composer Studio 3.1.
Figure 22(a) shows the execution time in terms of the number of bands used. The complexity of the system is directly related to the execution time. When the number of effective bands is increased, the complexity as well as the execution time are increased.

The computation complexity in terms of the number of target libraries is shown in Figure 22(b). The increasing rate of complexity is higher than the case shown in Figure 22(a) since the complexity of Step 3 is also increased as the number of libraries is increased.

The band selection is based on the relationship between backgrounds and libraries. The background samples represent the background area. Thus, the number of background samples is important for the effective band selection. Figure 23(a) shows the complexity in terms of the number of background samples. When the number of background samples is larger, the complexity of Steps 4 and 6 is increased. However, the total computation complexity is slightly increased.

The number of target samples is important for library refinement since the sample represents the detected image. Figure 23(b) shows the variation of computation complexity in terms of the number of background samples. 


\section{CONCLUSION}

This paper has presented spectral characterization for efficient image detection using hyperspectral processing techniques. We proposed an algorithm to reduce complexity and improve the library by using effective band selection and library refinement. The effective bands are heuristically selected for processing based on the contribution coefficient defined in this paper. The complexity of the proposed algorithm has been estimated in TMS320C6713 DSP. This approach has reduced the computation complexity. We have shown that for effective detection, only a small number of bands are needed.

\section{ACKNOWLEDGMENT}

This research is supported by the Ubiquitous Computing and Network (UCN) Project, the Ministry of Information and Communication (MIC) 21st Century Frontier R\&D Program in Korea.

\section{REFERENCES}

[1] T. Boggs and R. B. Gomez, "Fast hyperspectral data processing methods," in Geo-Spatial Image and Data Exploitation II, vol. 4383 of Proceedings of SPIE, pp. 74-78, Orlando, Calif, USA, April 2001.

[2] R. B. Gomez and A. J. Lewis, "On-board processing for spectral remote sensing," in ISPRS Special Session Future Intelligent Earth Observing Satellites (FIEOS '02), Denver, Colo, USA, November 2002.

[3] S. M. Chai, A. Gentile, W. E. Lugo-Beauchamp, J. Fonseca, J. L. Cruz-Rivera, and D. S. Wills, "Forcal-plane processing architectures for real-time hyperspectral image processing," Applied Optics, vol. 39, no. 5, pp. 835-849, 2000.

[4] G. A. Shaw and H. K. Burke, "Spectral imaging for remote sensing," Lincoln Laboratory Journal, vol. 14, no. 1, pp. 3-28, 2003.

[5] S. M. C. Nascimento, F. P. Ferreira, and D. H. Foster, "Statistics of spatial cone-excitation ratios in natural scenes," Journal of the Optical Society of America A, vol. 19, no. 8, pp. 1484-1490, 2002.

[6] R. C. Gonzalez and R. E. Woods, Digital Image Processing, Prentice-Hall, Upper Saddle River, NJ, USA, 2nd edition, 2002.

[7] W. H. Bakker and K. S. Schmidt, "Hyperspectral edge filtering for measuring homogeneity of surface cover types," ISPRS Journal of Photogrammetry and Remote Sensing, vol. 56, no. 4, pp. 246-256, 2002.

[8] M. L. Nischan, R. M. Joseph, J. C. Libby, and J. P. Kerekes, "Active spectral imaging," Lincoln Laboratory Journal, vol. 14, no. 1, pp. 131-144, 2003.

[9] M. K. Griffin and H. K. Burke, "Compensation of hyperspectral data for atmospheric effects," Lincoln Laboratory Journal, vol. 14, no. 1, pp. 29-54, 2003.

[10] G. P. Abousleman, M. W. Marcellin, and B. R. Hunt, "Hyperspectral image compression using entropy-constrained predictive trellis coded quantization," IEEE Transactions on Image Processing, vol. 6, no. 4, pp. 566-573, 1997.

[11] N. Keshava, "Distance metrics and band selection in hyperspectral processing with applications to material identification and spectral libraries," IEEE Transactions on Geoscience and Remote Sensing, vol. 42, no. 7, pp. 1552-1565, 2004.
[12] P. Bajcsy and P. Groves, "Methodology for hyperspectral band selection," Photogrammetric Engineering and Remote Sensing, vol. 70, no. 7, pp. 793-802, 2004.

[13] S. Kumar, J. Ghosh, and M. M. Crawford, "Best-bases feature extraction algorithms for classification of hyperspectral data," IEEE Transactions on Geoscience and Remote Sensing, vol. 39, no. 7, pp. 1368-1379, 2001.

[14] G. Girouard, A. Bannari, A. Harti, and A. Desrochers, "Validated spectral angle mapper algorithm for geological mapping: comparative study between quickbird and landsat-tm," in The 20th International Society for Photogrammetry and Remote Sensing Congress, pp. 599-605, Istanbul, Turkey, July 2004.

[15] R. Chassaing, Digital Signal Processing and Applications with the C6713 and C6416 DSK, John Wiley \& Sons, New York, NY, USA, 2005.

[16] Texas Instrument, "Datasheet of TMS320C6713B," November 2005, http://focus.ti.com/lit/ds/symlink/tms320c6713b.pdf.

Kyoung-Su Park received B.S. and M.S. degrees in electrical engineering from Chonbuk National University, South Korea, in 1999 and 2001, respectively, and M.S. degree in electrical and computer engineering from Stony Brook University - State University of New York in 2005. He is currently pursuing the Ph.D. degree at Stony Brook University. He was with Research Center of Hyosung Corporation, South Korea, from 2001 to 2002, where he was an RF Circuit and System Designer. His research interests include circuits for image processing and architecture optimization for high-performance DSP systems design.

Sangjin Hong received the B.S. and M.S. degrees in electrical engineering and computer science degree from the University of California, Berkeley. He received his Ph.D. in electrical engineering and computer science degree from the University of Michigan, Ann Arbor. He is currently with the Department of Electrical and Computer Engineering at State University of New York, Stony Brook. Before joining SUNY, he

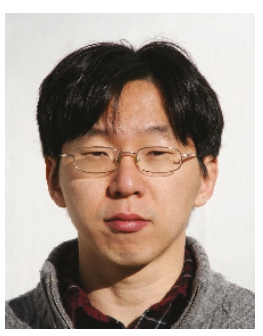
has worked at Ford Aerospace Corp. Computer Systems Division as a Systems Engineer. He also worked at Samsung Electronics in Korea as a Technical Consultant. His current research interests are in the areas of low-power VLSI design of multimedia wireless communications and digital signal processing systems, reconfigurable SoC design and optimization, VLSI signal processing, and lowcomplexity digital circuits. He served on numerous technical program committees for IEEE conferences. He is a Senior Member of IEEE.

Peom Park is a Professor in the Department of Industrial and Information Systems Engineering, Ajou University, Suwon, South Korea, also he is serving as Chief Executive Officer in HuminTec Co., Ltd. He got a Ph.D. degree from Iowa State University and worked in ETRI Electronics, Inc. in Korea. His research area is human-computer interaction in the applied IT technology with telemedicine, telematics, and ubiquitous lifecare system.

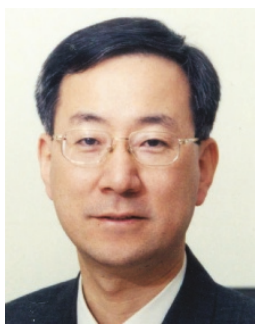


We-Duke Cho received a B.S. degree in 1981 from Sogang University, and his M.S. and Ph.D. degrees from Korea Advanced Institute of Science and Technology (KAIST) in 1983 and 1987. Currently, he is a Professor at the Department of Electronics Engineering College of Information Technology at Ajou University in South Korea. His research interests included ubiquitous computing/network, sensor network, post-PC

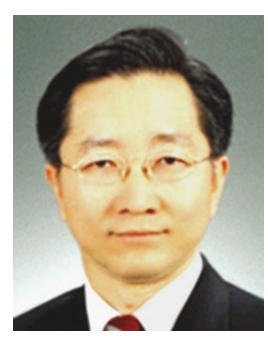

(next generation PC smart PDA), interactive DTV broadcasting technology, high-level home server and gateway, digital broadcasting and mobile convergence platform technology, and wireless network. 